\begin{tabular}{|c|c|c|c|}
\hline & $\begin{array}{l}\text { Wt. } \\
\text { Grams. } \\
\text { TreO } \\
\text { GranO }\end{array}$ & $\begin{array}{l}\text { Wt. } \mathrm{TeO}_{2} . \\
\text { Crams. }\end{array}$ & Atomic wt. of Te. \\
\hline $1 \ldots \ldots \ldots \ldots \ldots$ & 3.2059 & 2.6766 & $127 \cdot 4$ \\
\hline$\therefore \ldots \ldots \ldots, \ldots$ & $5 \cdot 1043$ & 4.2620 & $127 \cdot 4$ \\
\hline $3 \ldots \ldots \ldots \ldots \ldots \ldots$ & 4.6762 & 3.9046 & 127.4 \\
\hline \multirow[t]{2}{*}{$\therefore \ldots \ldots \ldots \ldots$} & 6.3670 & 5.3160 & $127 \cdot 4$ \\
\hline & - & $-1-2-1502$ & - \\
\hline
\end{tabular}

\title{
Conclusion.
}

III. The fractionation of crude material, with purification of the final fraction, is thus shown to be ineffective in securing any separation, as was quite to be expected. If the substance known as tellurium really is a complex body, the experiences which investigators too numerous to mention have had with it prove beyond question that the properties of the intermixed substances must be very closely alike, else a separation would long since have been obtained. A reaction, therefore, which if applied a sufficient number of times, might be capable of producing a separation upon purified tellurium, could hardly be expected not to be adversely affected by the presence of considerable quantities of various other elements.

It appears most probable to the writer that the chief cause of the failure of others, ${ }^{1}$ to get a separation has been a lack of practical appreciation of the closeness of the similarity between tellurium and its homolog. It will probably be found, if a complete separation is ever brought about, that in most reactions tellurium and its homolog behave identically, and in those reactions in which they differ, the differences will be slight. The problem is further complicated by the probability that the small differences which would be exhibited by the separated substances are either masked or nearly obliterated by their mutual influence, each upon the other, in the mixture.

The successful solution of the problem evidently depends primarily upon two factors : first, the securing of such a reaction as is most favorable to the separation of the similar constituents; and second, the continued application of this reaction for a sufficient length of time to produce an appreciable separation. In the writer's opinion, the hydrolytic method is at least as well suited to the purpose as any that have been tried and he is now engaged in giving it an exhaustive test. It is hoped that the work will be ready for publication within a reasonable time.

Chemical Iaboratory of Throop Polytechnic Institute, August 1, 1912.

\section{AN ELECTRICALLY HEATED MICROSCOPE SLIDE.}

BY F. G. COTTRELL.

Received June 18, 1912.

At the request of Dr. H. C. Biddle, and primarily to accompany his

'Cf. also Pellini, Atti accad. Lincei, 2I, I, 218-25. 
article on tannic acid and related compounds, ${ }^{1}$ the following description is given of an apparatus devised some years ago by the author more especially for the study of liquid crystals, but which will be found convenient wherever a particularly rapid and accurate adjustment of temperature under the microscope is desired.

The essential feature of the method lies in depositing a uniform film of platinum over the glass slide, which is to carry the object, so thin as not to seriously reduce its transparency but still forming a continuous conductor for the electric current. By connecting this film in series with a suitable theostat to a convenient source of current, such as an ordinary I 10 or 220 volt lighting circuit, any temperature from that of the room to almost the melting point of the glass may be rapidly established.

To minimize the danger of cracking under the heat, especially for high temperatures, the glass slips to be coated should be made quite small and of thin glass. It has proved most convenient to make these from the thin quality (about 0.6 to $0.8 \mathrm{~mm}$.) of $\mathrm{I} \times 3$ inch microscope slides by cutting the latter crosswise in $1 / 2$ inch widths and rubbing down these cut edges on fine emery cloth or the finest carborundum powder to eliminate any incipient crack or strain left on the edge from the cutting.

Both ends of these little $1 / 2 \times$ I inch slides are then painted for say $1 / 4$ inch with gold paint such as is used for decorating porcelain. If this is not at hand, a good substitute is a collodion solution to which a small amount of either gold or platinum chloride has been added. In this case, the ends of the slide may be dipped in the collodion to the desired depths and the adhering film allowed to dry in the air. The slide is in either case next placed over a Bunsen burner and the heat gradually raised until the organic matter is completely burned out and a brilliant metallic surface left, which may be thickened, if necessary, by repeated paintings and burnings. If thus thoroughly fired, this is tightly adherent and will stand considerable handling. These gilded or platinized ends of the slides serve as terminals for the still thinner film subsequently to be spread between them and which serves as the electrical resistance for heating.

Unfortunately this very simple method of gilding or platinizing conductive coatings on glass, while entirely adequate for these terminal strips, fails to produce sufficiently uniform thin coatings to be used for the resister, so it is necessary to resort for this to the discharge (Zerstaubung) from a platinum cathode in high vacuum, a method familiar for many years to physicists.

A convenient and easily assembled apparatus for carrying this out consists of a stout test tube, $A$ (Fig. I) fitted with rubber stopper through

${ }^{1}$ This JOURNaL, 34, 918 (1912). 
which pass tube $B$ for connection to the vacuum pump, an aluminum wire, $C$, for an anode and a glass tube, $D$, through the inner end of which
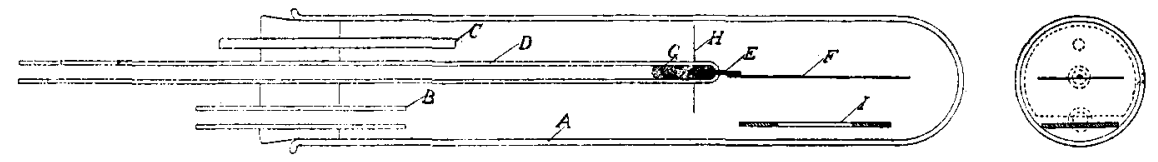

Fig. I.-One-fifth natural size.

is sealed a platinum wire, $E$, to the free end of which is welded the platinum plate $F$, to serve as a cathode. This platinum wire and plate should be stiff enough to retain their position without bending if the apparatus is shaken. Electrical connection to the cathode is easily made by pouring a few drops of mercury into the glass tube and shaking it down into the end of the tube as at $G$. The negative wire from the induction coil may then be thrust down the tube into this mercury. A mica plate, $\boldsymbol{H}$, may also be slipped over the cathode tube nearly up to its end to screen the inlet end of the outer tube from deposit and thus facilitate the examination of the glass slip $I$ as platinizing proceeds.

This strip, with its ends gilded or platinized as previously described, is slipped into tube $A$ and the stopper inserted and all joints thoroughly painted with molten wax, made preferably by dissolving io $\%$ to $20 \%$ of pure gutta percha in molten beeswax. The vessel is then exhausted with a mercurial air pump or other means capable of producing a vacuum of say $0.01 \mathrm{~mm}$. of mercury. The attainment of the required vacuum is sufficiently indicated by the appearance of the well known green fluorescence of the glass when the current from the induction coil is applied. This coil should be capable of giving at least a one inch spark in air.

Within less than a minute after the current has been applied, a very perceptible darkening of the walls of the test tube and the surface of the glass slip should become apparent. If the apparatus is connected to the pump by a thick-walled rubber tube, it is an easy matter to shake the glass slip back and forth past the mica screen and thus examine it through the clear walls of the left hand end of the tube. As soon as a slight but uniform darkening of the glass between the gilded tips is perceptible, the slip is finished and may be removed and another treated. After preparing and testing a few slides, it is easy to judge by the eye the best thickness of deposit. By sufficiently extending the time or strength of the discharge in the vacuum tube, brilliant and entirely opaque mirrors may be obtained. In fact this method may be employed to produce the heavy deposits on the ends of the slides in place of the gilding process above described. In this case the central portion of the slide is to be protected from deposit by wrapping with tin foil. 
A convenient form of holder for these slips is shown in Fig. 2. On an ordinary $I \times 3$ inch microscope slide are cemented (preferably after roughening the glass surface with emery or carborundum) the two small binding posts $B$, with large flat bases, carrying light metal springs, $C$. The gilded ends of the small glass slips are wound

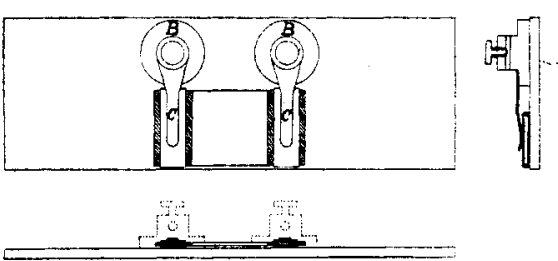

Fig. 2.-One-fifth natural size. round with several thicknesses of tin foil and then slipped under these springs as shown in the figure. The whole slide may now be handled in the usual way, only taking care not to short-circuit the heater through the spring clips on the microscope stage if these are used.

A convenient form of rheostat for use with this apparatus consists of a glass cylinder about $\mathrm{I}$ inch in diameter by 4 inches high on a substantial foot. Through a cork stopper pass two glass-stemmed platinum electrodes similar to that described as cathode in the platinizing tube except that the platinum sheet may be much smaller or event entirely omitted. One of these should reach to the bottom of the cylinder, the other sliding easily through the cork in order to allow adjusting the distance between their. tips.

The cylinder is filled with distilled water to which a drop of dilute sulfuric acid is added. For temperatures on the slide only slightly above that of the room, even higher dilution may be needed.

With nonconductors or very poor conductors as in the case of most organic compounds, the material may be examined to best advantage by placing it directly upon the platinized surface of the glass, in which case the temperature response to adjustments of the rheostat is practically instantaneous and can be controlled with the greatest delicacy while observing. With electrolytes this is not usually practical, but in this case a slip of thin cover glass may be first laid upon the heating surface. The temperature response then though still rapid is markedly slower than in the other case.

The apparatus is particularly useful in studying melting and recrystallization phenomena, as any individual crystal may be watched and its melting or crystallization instantly stopped and held constant or reversed at will.

No attempt has been made in the use of the apparatus thus far to measure the temperature, but it would seem this might be done by measuring the current flowing through the platinum with a galvanometer while measuring drop in potential across the platinum film by the Poggendorf compensation method and from these data determining the resistance of the film itself which, if pure platinum is used, should be directly pro- 
portional to the absolute temperature. While there might be some minor corrections to apply, these could be experimentally determined by observations on substances of known melting point.

JWREEXEY, CALXRORNIA

\section{THE RELATIVE STABILITY OF PRIMARY CADMIUM POTASSIUM IODIDE AND ITS APPLICATION IN THE DETER- MINATION OF OZONE.:}

Bi Charles Baskerville and W. J. Crozier.

Received July 12, 1912.

Of the several methods which have been proposed for the determination of ozone, that of Schönbein, which consists in estimating the iodine liberated from metallic iodides, has been quite generally employed, although it is open to a number of serious objections. It is well known that when acid potassium iodide solution is employed, the potassium iodide-starch reaction is neither a qualitative nor a quantitative indication of the presence or amount of ozone; and that various agencies influence the liberation of iodine from the acidified potassium iodide solution, while chlorine, bromine and nitrites (frequently found in the air) also give the reaction. These influences, of an order of magnitude safely disregarded in other analytical work, must be dealt with. With regard to the reliability of neutral metallic iodide solutions, Lechner ${ }^{2}$ maintained that unsatisfactory results were obtained when determining ozone with the aid of neutral potassium iodide solutions, as large quantities of iodine vapor were liberated. This is directly contrary to the results obtained by Ladenburg and Quasig,, who found that neutral solutions only give correct results, and that acid solutions give no sharp end-point and the results are too high. ${ }^{4}$ They obtained good results, but used, apparently, only ozone of a high state of purity.

Baskerville and Hamor ${ }^{5}$ have pointed out the objections to which potassium iodide is open as a reagent for hydrogen dioxide, and the authors have found that as a test for ozone, potassium iodide is subject to the same limitations, and, generally, even to a greater degree. Baskerville and Hamor found that since primary cadmium potassium iodide $\left(\mathrm{CdKI}_{3} \cdot \mathrm{H}_{2} \mathrm{O}\right)$ is unaffected by the presence of any likely amounts of acids in ethyl ether, and is much more stable in light than potassium iodide in solutions of comparable strength, its use is preferable for the detection of peroxidized compounds in ether. The authors have extended the observa-

Read before the Eighth International Congress of Appiied Chemistry, New York, Sept, 1912 .

Z. Elektrochem. I7, 412.

Ber., 34, I 84 (1901).

- Our experiments would tend to confirm these findings.

5. Ind. Eng. Chem., 3, No. 6 (19II). 